\title{
Quasi-static Microscanner with Linearized Scanning for an adaptive 3D-Lasercamera
}

\author{
Th. Sandner ${ }^{1}$, Th. Grasshoff ${ }^{1}$, M. Schwarzenberg ${ }^{1}$, H. Schenk ${ }^{1}$ \\ ${ }^{1}$ Fraunhofer Institute for Photonic Microsystems (IPMS), AMS, Dresden, Germany, \\ thilo.sandner@ipms.fraunhofer.de
}

\begin{abstract}
This paper presents a gimbaled MEMS scanning mirror (MSM) especially developed for adaptive raster scanning in a novel 3D ToF laser camera. Large quasi-static deflections of $\pm 10^{\circ}$ are provided by vertical comb drives in vertical direction in contrast to resonant horizontal scanning of the $2.6 \times 3.6 \mathrm{~mm}$ elliptical mirror at $1600 \mathrm{~Hz}$ and $80^{\circ}$ optical scan range. For position feedback piezo-resistive position sensors are integrated on chip for both axes. To guarantee the full reception aperture of effective $5 \mathrm{~mm}$ a synchronized driven MEMS scanner array - consisting of five hybrid assembled MEMS devices - are used in a novel 3D ToF laser scanner enabling a distance measuring rate of $1 \mathrm{MVoxel} / \mathrm{s}$ and an uncertainty of ToF distance measurement of $3 \ldots 5 \mathrm{~mm}$ at $7.5 \mathrm{~m}$ measuring range for a gray target.
\end{abstract}

Key words: Optical MEMS, linearized raster scanning, vertical comb drive, LIDAR, 3D sensing, ToF time-of-flight distance measurement

\section{Introduction}

Laser scanners are widely used for time of flight (ToF) 3D-distance measurements systems. In comparison to focal plane array based ToF 3Dcameras laser scanners have an advantage of higher measurement accuracy due to scanning principle, because only single measuring points are illuminated sequentially within the scanned FOV. Hence, the LIDAR detector collects light of signal- \& background (noise) in short time only from a small measured area of the target reducing significantly the influence of background (noise). Also a nearly unlimited number of 3D data (voxels) can be measured with high measuring rates of up to $1 \mathrm{Mio}$ voxel/s and low measurement uncertainty of typically $3 . .10 \mathrm{~mm}$. One the other hand, traditional laser scanners for 3D distance measurement involve expensive, heavy and large rotating or vibrating mirrors for light deflection of the scanning ToF (time of flight) distance measurement. Typically, the precision of ToF distance measurement is limited by the amount of signal light available at the detector. Hence, a scanning mirror with large aperture is required for LIDAR systems to collect small amounts of light reflected or scattered by the measured target. Traditionally, polygon scanner are used, but for high scanning speeds (e.g. > 30000 turns/min) large efforts (e.g. air bearings) are required to reduce abrasive wear resulting in heavy $(>10 \mathrm{~kg})$ and cost intensive scanning units. Its replacement by micromechanical scanning mirrors, which have the benefits of high scanning speed, low weight, high mechanical reliability (e.g. no friction, high shock resistance), is not straightforward, since a large mirror aperture of the receiver optics must by guaranteed in addition to sufficiently large optical scan angles $\left(>40^{\circ}\right)$ and high scan frequency of more than $100 \mathrm{~Hz}$. Contrary, the aperture of a single MEMS scanning mirror is limited to small values of typically $1 \ldots 3 \mathrm{~mm}$ diameter due to the dynamic mirror deformation. To overcome the mentioned problems, Fraunhofer developed the concept for a MEMS-based LIDAR based on an array of identical synchronized driven MEMS elements [1]. A first prototype - developed for a phase shifting distance measuring system [2] - were limited to resonant 1D scanning at $250 \mathrm{~Hz}$ and $60^{\circ} \mathrm{FOV}$ of a monolithic MEMS array with $80 \%$ optical filling factor, total aperture of $334.2 \mathrm{~mm}^{2}$ consisting of $2 \times 7$ MEMS mirrors driven synchronized to a separate sending mirror [3].

\section{Concept of an adaptive 3D TOF laser camera}

Now, we extend the MEMS based LIDAR to the novel concept of an adaptive 3D ToF laser camera with foveation properties [4] to allow e.g. future autonomous robots to better interact with their surroundings. The 3D sensor concept of foveation - that is acquiring distance images at video like frame rates with coarse spatial resolution, rapidly detecting regions of interest (ROI), and then concentrating further image acquisition on these ROls with adaptive scanning - requires a challenging $2 \mathrm{D}$ scanning device with quasi-static actuation, large effective aperture $\geq 5 \mathrm{~mm}$, and large FOV $>60^{\circ}$. The best technical compromise of the fast 
adaptive scanning unit were found in a quasistatic / resonant raster scanning MEMS mirror to (i) meet opposite requirements of fast scanning $(>1000 \mathrm{~Hz})$, large optical scan range combined with large single mirror aperture and (ii) to enable partially foveation by adaptive vertical scanning (see Fig. 1).

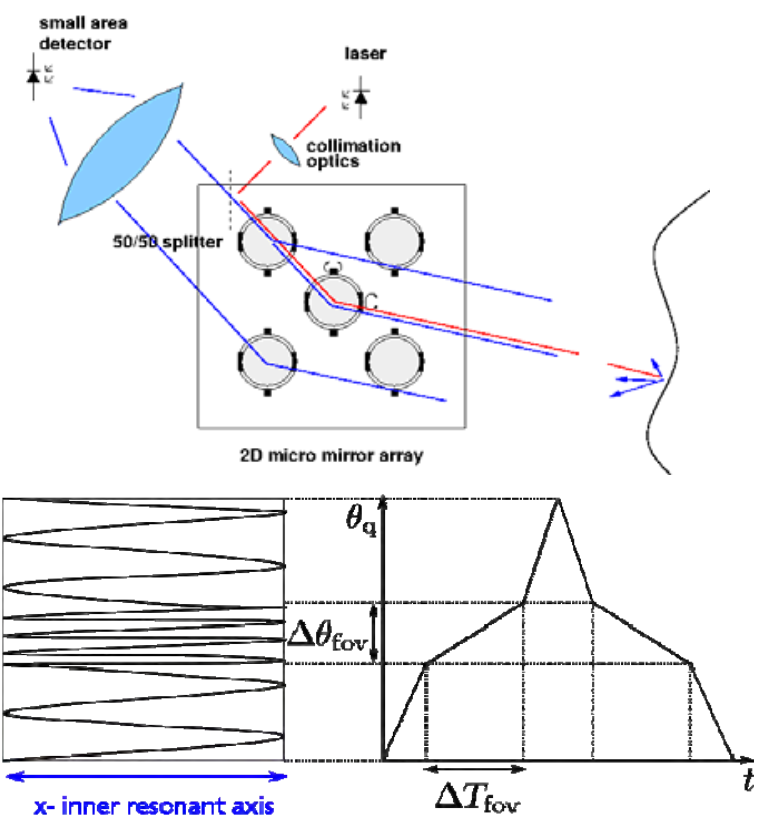

Fig. 1. TACO Concept of adaptive MEMS based 3D-laser camera: (above) Schematic optical setup using a synchronized 2D-MSM array to provide sufficient reception aperture, (below) $2 D$ raster scan with adaptive vertical scanning enabling partially foveation with higher resolution in ROl's $\Delta \theta_{\text {fov. }}$.

To provide the effective reception aperture of $5 \mathrm{~mm}$ a MSM array consisting of five hybrid assembled MEMS scanning mirrors are precisely synchronized operated in respect to the sending mirror of the ToF laser scanner. The target specifications of the 3D camera are summarized in the following table:

Tab. 1: Target specification of adaptive $3 D$ camera

\begin{tabular}{|c|c|c}
\hline Parameter & Unit & value \\
\hline Distance min & $\mathrm{cm}$ & $20(10)$ \\
\hline Distance max & $m$ & $7.5(15)$ \\
\hline FOV horizontal & $\circ$ & $60(80)$ \\
\hline $\begin{array}{c}\text { FOV vert.: qs } \\
\text { (dyn.) }\end{array}$ & $\circ$ & $40(60)$ \\
\hline $\begin{array}{c}\text { Beam divergence } \\
\text { 3D-measuring rate }\end{array}$ & Voxel/s & 2 @1550nm \\
\hline $\begin{array}{c}\text { Distance accuracy } \\
\text { Frame rate; } \\
\text { variable }\end{array}$ & frame/s & $1 \ldots 140$ \\
\hline & $25 @$ HQVGA \\
\hline
\end{tabular}

\section{LinScan concept for quasi-static actuation}

Electrostatic resonant MSMs of Fraunhofer IPMS are one of the most recognized MEMS scanner devices proven for reliable industrial fabrication [5]. However, these 2D MSMs with in-plane comb drives are limited to resonant operation at fixed frequency or frequency ratio. Thus, the scan trajectory is fixed and depends on the amplitude and the frequency ratio predefined by the MEMS design.

Recently, IPMS extended its scanner technology to quasistatic actuation using threedimensional vertical out-of-plane comb drives. The novel device concept for vertical 3D comb drives - named LinScan - was realized by IPMS as system-in-package device and assembly [6]. Geometry of activated vertical combs are geometrically dependent on the solid body mechanism used to deflect the fixed electrode from the in plane fabrication position to the desired out of plane position. Depending on the design of solid body mechanism both staggered (SVC) or more efficient angular (AVC) comb drives can be realized within the same process [7]. In contrast to state of the art for MSM with 3D comb drives [8][9] our approach enables larger scan angles up to $\pm 11^{\circ}$ combined with simplified and reliable fabrication with high flexibility of drive geometry only by design without technological changes. The technology approach is reported in more detail in [10].
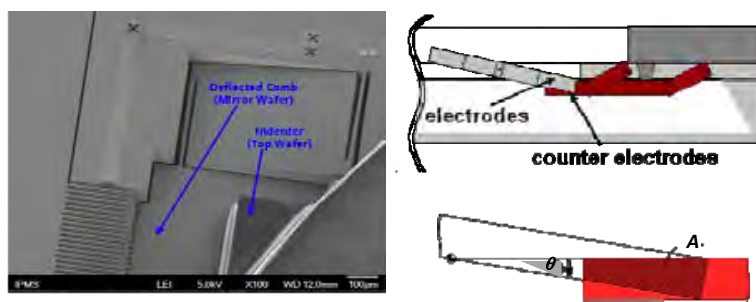

Fig. 2. System in package concept for vertical comb drives; schematic setup (left) SEM image of activated SVC driving electrode (right).

\section{MEMS device for 2D raster scanning}

The 2D raster scanning MEMS mirror was especially developed for the novel system concept of an adaptive 3D-ToF laser camera shown in Fig. 1. The 2D-MEMS device consists of a resonant inner mirror gimbal suspended within a quasi-static actuated frame. Whereas the inner mirror is actuated in parametric resonance by in-plane vertical comb drives, 3D out-of-plane vertical comb drives are used for quasi-static actuation of outer frame. In Fig. 3 a microscopic photograph of the 2D-MEMS is shown captured via the free aperture of the cover substrate used to activate the $3 D$ vertical comb drives (see also Fig. 2). Details of the quasi-static frame and the basic electrical configuration of the staggered vertical comb 
(SVC) drive used for quasistatic actuation are also shown in Fig. 3. For a SVC drive the movable and fixed driving electrodes are out-ofplane shifted for rest position of the torsional spring suspended frame. The driving voltages (U1, U2) are applied separately to the two fixed driving electrodes - whereas the movable electrode is connected to ground - enabling quasistatic forward and backward actuation of the movable frame.

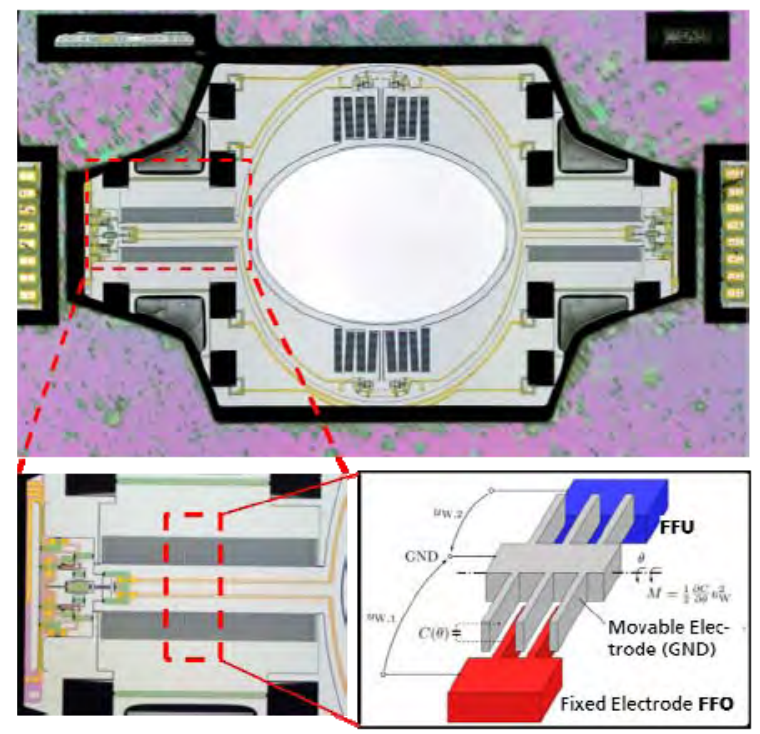

Fig. 3. Photograph of quasistatic / resonant $2 D$ MSM raster scanning mirror; (below) details of quasistatic drive and electrical driving schema of staggered vertical comb (SVC) drive.

The target specification of the resonant / quasistatic 2D-MEMS scanner is shown in Tab. 2.

Tab. 2: Specification of 2D-MEMS scanner design

\begin{tabular}{|c|c|c|c}
\hline Parameter & Unit & Res. mirror & Qs frame \\
\hline Mirror aperture & $m m$ & $2.6 \times 3.6$ & - \\
\hline dyn. def. $\sigma_{R M S}\left(\sigma_{\text {max }}\right)$ & $\mathrm{nm}$ & $23.4(110) @ 22.5^{\circ}$ \\
\hline Eigen frequency & $\mathrm{Hz}$ & 1600 & 125 \\
\hline$M S A_{\text {nom }}$ & $\circ$ & 20 & $10 @ D C$ \\
\hline$M S A_{\max }$ & $\circ$ & 24 & 15 \\
\hline$U_{\text {drive }} @ M A_{\text {nom }}$ & $V$ & 160 & $150 \mathrm{DC}$ \\
\hline chip size & $\mathrm{mm}$ & \multicolumn{3}{|c}{$10.6 \times 7.0$} \\
\hline
\end{tabular}

The torsional spring suspension of the $4.6 \times 4.9 \mathrm{~mm}^{2}$ large and $75 \mu \mathrm{m}$ thick elliptical frame was designed for the quasi-static equilibrium of spring torque and electrostatic driving torque generated by the optimized SVC electrodes. For a static mechanical deflection of $\pm 10^{\circ}, 125 \mathrm{~Hz}$ Eigen-frequency results for the frame at limited DC voltage of $150 \mathrm{~V}$. For dynamic actuation from DC to $125 \mathrm{~Hz}$ the frame can achieve larger mechanical scan angles up to $\mathrm{MSA}= \pm 15^{\circ}$. Nevertheless, for horizontal scanning larger scan angles up to MSA $= \pm 17 \ldots 20^{\circ}$ are required at $1600 \mathrm{~Hz}$ scan frequency. Due to limited driving efficiency of electro static actuation these requirements are not achievable with a quasistatic actuation of inner mirror, instead an operation in parametric resonance is essential. An elliptical mirror aperture of $2.6 \times 3.6 \mathrm{~mm}^{2}$ where chosen to provide a beam divergence of $\leq 1 \mathrm{mrad}$ at $45^{\circ}$ angle of incidence. To guarantee $\leq \lambda / 10$ optical flatness even for the relative large mirror an advanced mirror suspension with distributed spring [5] was used to minimize the dynamic mirror deformation to $\leq 110 \mathrm{~nm}$ (peak-peak at $M S A= \pm 22.5^{\circ}$ ) which is sufficient for the collimated laser beam of $\lambda=1550 \mathrm{~nm}$. For real time feedback driving control piezo-resistive position sensors are integrated on chip for both scanning axis. Therefore two bending beams used as mechano-electrical transducers and wired to form a resistive WHEATSTONE half bridge - are added symmetrically to the anchor point of the torsional string suspensions.

\section{Experimental results}

In figure 4 the static deflection characteristics of the quasi-static frame - i.e. the mechanical tilt angle versus DC driving voltage - measured in static equilibrium are shown for positive and negative scan direction. A symmetric but progressive driving characteristic with maximal mechanical deflection of $\mathrm{MSA}= \pm 10^{\circ} @ 150 \mathrm{~V}$ $\mathrm{DC}$ driving voltage is obvious.

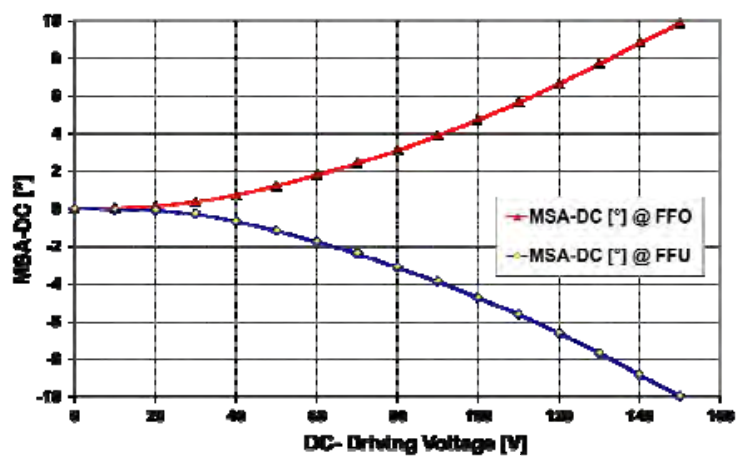

Fig. 4. Static deflection characteristic of the quasistatic frame; mechanical tilt angle vs. driving voltage plotted for positive and negative scan direction.

The frequency response characteristics of inner mirror - measured in parametric resonance for varied pulse driving voltages (30...125V) and $50 \%$ duty cycle - are exemplary shown in Fig. 5. For a given voltage the maximal amplitude is achieved at the unstable point of resonance. Under normal ambient conditions typically a mechanical angle of $\mathrm{MSA}= \pm 17 \ldots 18^{\circ}$ was achieved at $125 \mathrm{~V}$ pulse driving voltage. For a slightly reduced ambient pressure of $30 \mathrm{kPa}$ up 
to $\mathrm{MSA}= \pm 24^{\circ}$ have been demonstrated. To achieve a stable operation at Eigen frequency resulting in maximal amplitude the mirror has to operate in synchronized operation were the phase of pulse driving voltage must to be controlled by the phase of mirror oscillation [5]. The inset of Fig. 5 shows the dependency of mechanical deflection and resonance frequency vs. driving voltage in synchronized operation mode. At $160 \mathrm{~V}$ a mechanical tilt angle up to $\pm 20^{\circ}$ were achieved in normal ambient. On the other hand the Eigen frequency is affected by fabrication tolerances of the DRIE trench etching process. Hence, to realize a 3D camera with synchronized 2D-MEMS scanner array where all single MEMS devices have to oscillate with same frequency, phase and amplitude related to the central sending mirror a MEMS driving in parametric resonance at equal driving frequency is essential. Therefore, all MEMS devices of the array have to guarantee an overlapping frequency band at target scan amplitude, where amplitude can be adjusted individual for each scanning mirror by driving voltage and phase by delaying the pulse voltage.

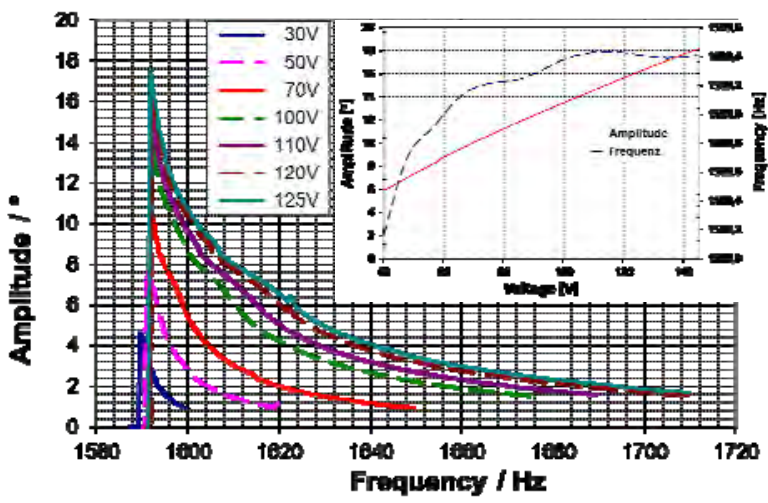

Fig. 5. Frequency response curve of resonant inner axis in parametric resonance measured for various driving voltages. Inset shows characteristics of synchronized operation mode: mechanical deflection and resonance frequency vs. driving voltage.

The frequency bandwidth of an individual MEMS mirror - measured in parametric resonance at varied pulse driving voltages limited to $120 \mathrm{~V}$ - is shown in Fig. 6.

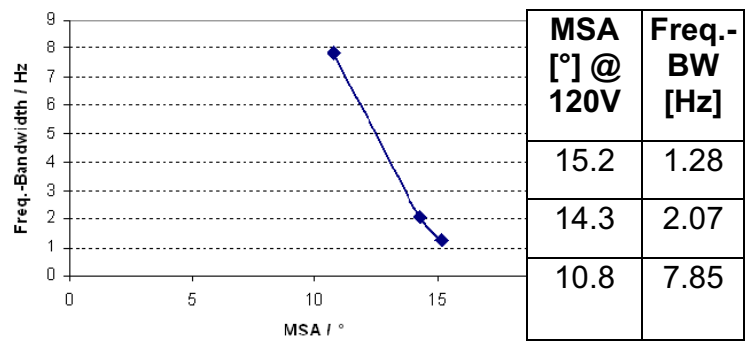

Fig. 6. Frequency bandwidth of inner mirror vs. mechanical scan angle measured in parametric resonance for pulse driving voltages limited to $120 \mathrm{~V}$.
A decrease of frequency bandwidth with increasing scan angle is obvious. Only $1.3 \mathrm{~Hz}$ frequency bandwidth were measured at MSA $= \pm 15.2^{\circ}$. Nevertheless, to enable a synchronized operation of multiple MEMS elements with reasonable yield and coast a hybrid assembly were chosen to build up the MEMS scanner array instead of a monolithic MEMS array originally used in [1][3]. The deviation of resonance frequency was measured for several wafers. In the worst case a maximal frequency difference of $1.03 \mathrm{~Hz}$ were measured, whereas the frequency difference of selected MEMS devices is typically only $0.04 \ldots 0.15 \mathrm{~Hz}$ sufficiently smaller in comparison to the frequency bandwidth of about $1.3 \mathrm{~Hz}$. For synchronized operation of multiple MEMS elements also thermal induced frequency changes must be compensated by driving control. For the 3D laser camera a fiber amplified pulsed laser with about $P_{C W}=1.4 \mathrm{~W}$ laser power is used. To investigate the effect of absorbed laser irradiation the frequency and amplitude change of the resonant inner mirror were measured in synchronized driving mode at constant driving voltage for varied $\mathrm{CW}$ power using a diode laser heating the mirrors backside (see figure 7). A sufficiently small frequency decrease of $\Delta \mathrm{f}_{0}=-0,3 \mathrm{~Hz}$ and an amplitude change of only $\Delta \theta=+0,007 \%$ were measured for an equivalent CW laser power of $1.4 \mathrm{~W} @$ $\lambda=1550 \mathrm{~nm}$ not crucial for synchronization.

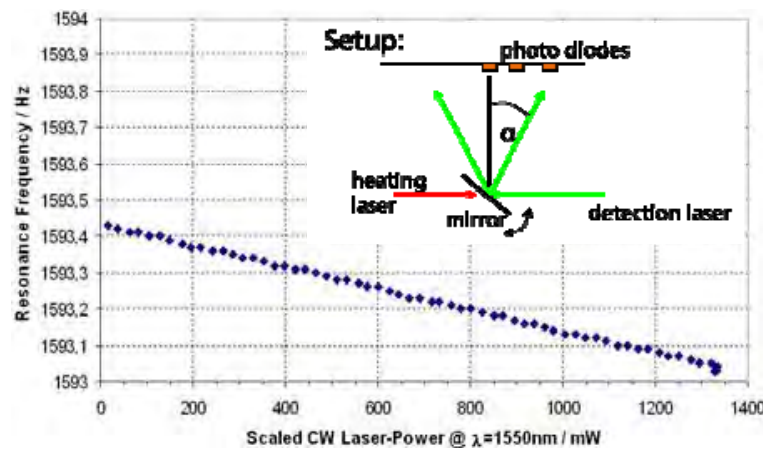

Fig. 7. Influence of absorbed laser irradiation on resonant frequency of resonant inner axis measured in synchronized mode at constant driving voltage.

For prospective real-time feedback control piezo-resistive position sensors are integrated on chip for both scanning axis, but currently not taken into account for the open loop control approach currently used in the 3D camera. Fig. 8 depicts the geometry of the piezoresistive position (PZR) sensor exemplary shown for the quasi-static frame. In the chase of the quasistatic frame the PZR position can be configured by external wiring to either a full or half WHEATSTONE bridge; whereas the PZR sensor of the inner mirror was implemented as half WHEATSTONE bridge due to limited number 


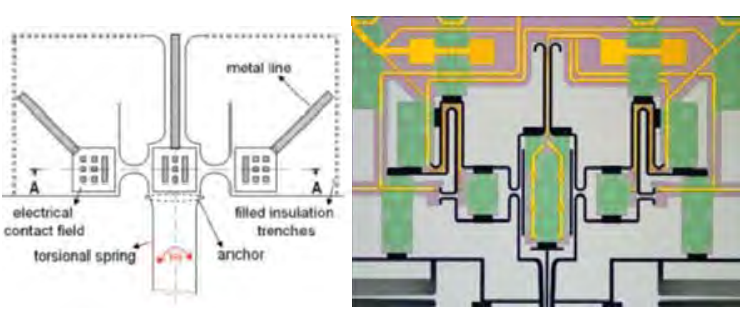

Fig. 8. Piezo-resistive position sensor of quasistatic frame; shown is schematic sensor geometry (left) and microscopic photograph (right) of half WHEATSTONE bridge PZR sensor.

of electrical potentials which can be wired to the gimbal suspended inner mirror. The PZR sensor characteristic of quasi-static frame, measured for a full WHEATSTONE bridge is shown in Fig. 9. A good linearity and sufficient sensitivity is obvious, promising for a dynamic closed loop driving control of the quasistatic frame required to achieve the adaptive scanning properties of the foveated 3D camera.

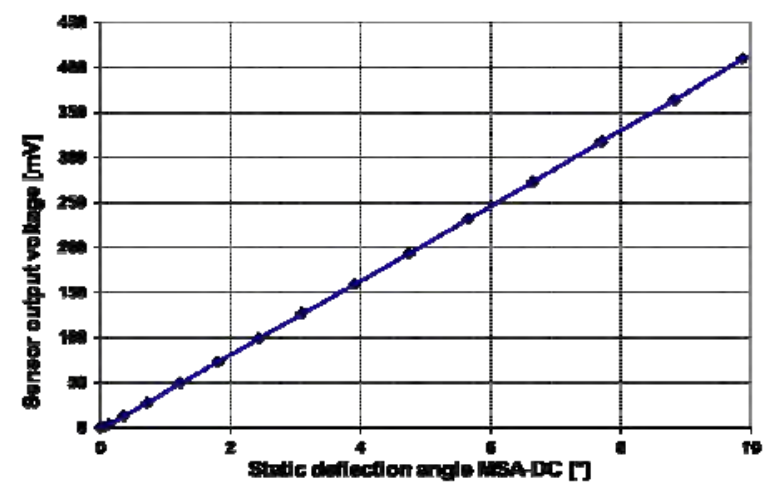

Fig. 9. Characteristic of piezo-resistive position sensor of quasi-static frame, measured for a full WHEATSTONE bridge PZR sensor.

Even though the quasistatic frame can provide substantial static tilt up to $\pm 10^{\circ}$ due to its SVC comb drives it still represents a high $Q$ spring mass system only very lowly damped by the surrounding viscous gas. In Fig. 10 the measured step response of the quasistatic axis is exemplarily shown for an angular step from rest position to $\mathrm{MSA}=5^{\circ}$. Low damping resulting in large settling time $>1$ s and large oscillations at Eigen frequency of $125 \mathrm{~Hz}$ are obvious.

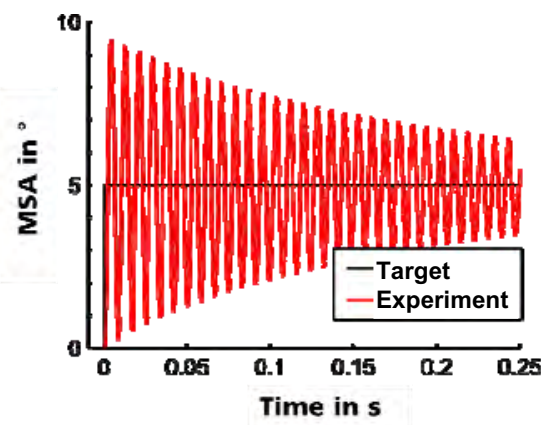

Fig. 10. Steep response to $5^{\circ}$ of the quasistatic axis
This makes the driving control a challenge requiring a dedicated driving control to compensate the poor Eigen dynamics of the MSM. The adaptive 3D-TOF camera needs vertical scan trajectories with variable linearized scanning below scanner Eigen frequency of $125 \mathrm{~Hz}$. Typically (i) symmetric triangular scanning patterns at $10 \mathrm{~Hz}$ or (ii) symmetric linear scan patterns with reduced scanning velocity are required for higher resolved ToF measurement in ROl's. In addition the maximum deviation of temporal angular scan position is limited to only $0.1^{\circ}$ for all five scanning mirrors over the entire scan range due to the small FOV of the fiber coupled detector optics to guarantee the full effective reception aperture of the adaptive 3D TOF camera. Several methods for open loop MEMS driving control were tested. The best results were achieved by using a flat based OL control [12], some experimental scan trajectories are shown in Fig. 11 and 12 for different scan patterns successfully verifying and demonstrating the potential of adaptive scanning.
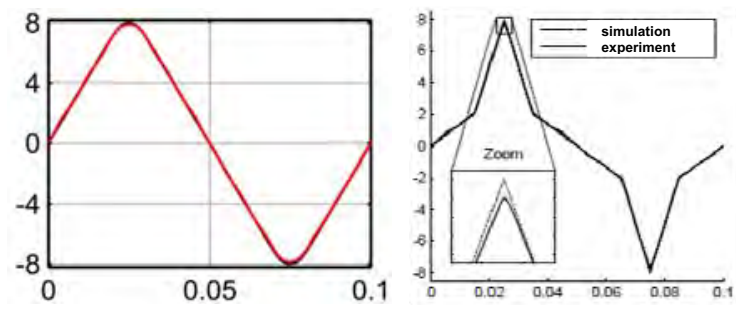

Fig. 11. Measured trajectories of quasistatic axis with linearized scanning at $10 \mathrm{~Hz}$ with flat $\mathrm{OL}$ control; triangular (left) stepped linearized for foveation (right)
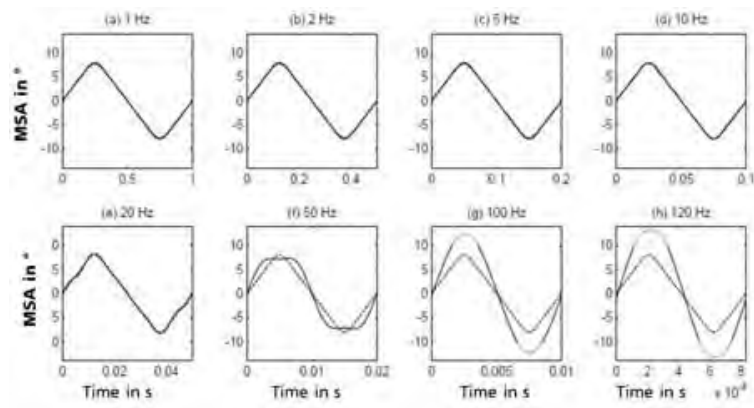

Fig. 12. Dependency of linearized scan trajectory on scan frequency varied from $1 . .120 \mathrm{~Hz}$

\section{System integration of MEMS scanner array}

To provide the full $5 \mathrm{~mm}$ effective reception aperture of the ToF camera five 2D raster scanning MEMS mirrors - selected by frequency - are hybrid assembled to a MEMS array, where all scanning mirrors are precisely synchronized operated in respect to the sending mirror of the ToF laser scanner. The MEMS array is built in a symmetric coaxial assembly of four peripheral reception mirrors around the centered sending mirror. The 
schematic setup of the optical scan head is shown in Fig. 11. Due to the limited optical filling factor $<20 \%$ of the 2D MSM optical guiding prisms are used to transfer the light collected by of peripheral MEMS mirrors near to the optical axis enabling to focus the received light to the fiber coupled APD detector needing a fiber core diameter of only $100 \mu \mathrm{m}$. Details of the optical sensor design can be found in [11].
The pulsed ToF distance measuring system use a fiber amplified laser of $P_{C W}=1.5 \mathrm{~W}$, $\lambda=1550 \mathrm{~nm}$ and $1 \mathrm{MHz}$ pulse repetition rate resulting in a distance measuring rate of $1 \mathrm{MVoxel} / \mathrm{s}$ and a measurement uncertainty of $3 . .5 \mathrm{~mm}$ at $7.5 \mathrm{~m}$ measuring range enabling e.g. $3 \mathrm{D}$ images with $1 \mathrm{Mpixel}$ per second or 10 frames of $100 \mathrm{k}$ pixel per second, respectively, over a $40^{\circ} \times 60^{\circ}$ (potentially $60^{\circ} \times 80^{\circ}$ ) FOV.
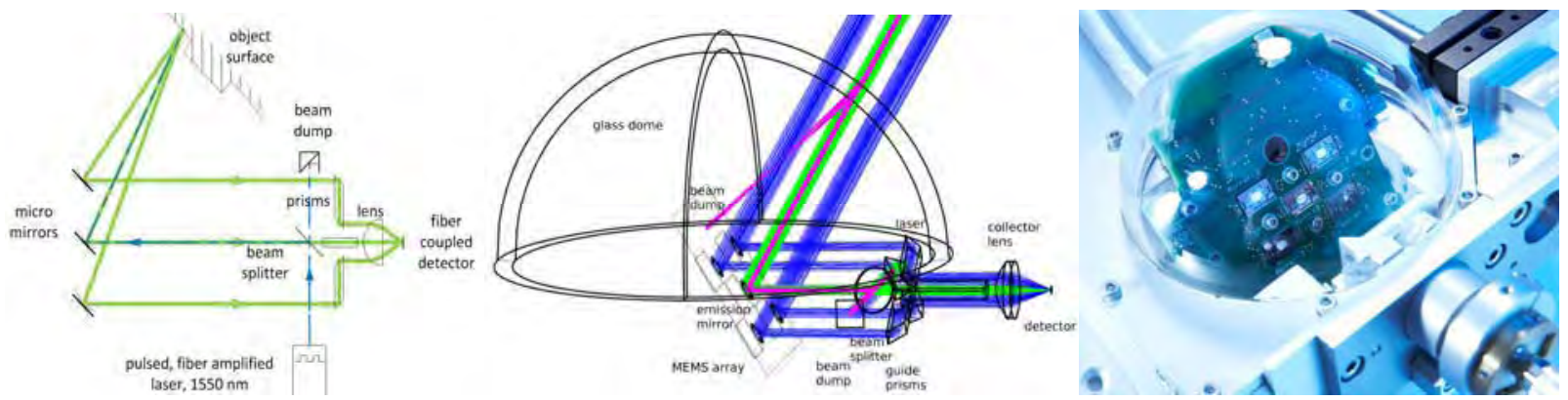

Fig. 13. System integration of hybrid assembled 2D-MEMS scanner array: (left) schematic optical setup, (middle) optical scan head of 3D camera developed by Fh-IPM [11], (right) photograph of assembled optical scan head.

\section{Conclusion}

A gimbals suspended quasistatic / resonant 2Draster scanning MEMS mirror were developed for use as fast scanning unit of a novel adaptive 3D-TOF laser camera. Adaptive linearized scanning in vertical direction is realized by quasistatic SVC comb drives enabling a 3D camera with partial foveation properties to allow e.g. future autonomous robots to better interact with their surroundings. Static tilt angles of MSA $= \pm 10^{\circ}$ and variable scan frequencies from DC...125 Hz were experimentally verified not possible with a common resonant MSM. Resonant horizontal scanning at $1600 \mathrm{~Hz}$ guaranties a large optical scan range of up to $80^{\circ}$ even for the $2.6 \times 3.6 \mathrm{~mm}$ large single mirror. To provide the full $5 \mathrm{~mm}$ effective reception aperture - required by the ToF camera - five frequencies selected 2D MEMS raster scanning mirrors were hybrid assembled and driven in synchronized 2D operation with respect to the sending mirror. These enable the best technical compromise of opposite requirements: partially foveation, fast scanning speed, large scan range, large effective aperture, high yield and low cost. The novel adaptive 3D ToF camera provides a distance measuring rate of $1 \mathrm{MVoxel} / \mathrm{s}$ and an uncertainty of ToF distance measurement of $3 \ldots 5 \mathrm{~mm}$ at $7.5 \mathrm{~m}$ measuring range enabling e.g. 3D images with $1 \mathrm{Mpixel}$ per second or 10 frames of $100 \mathrm{kpixel}$ per second, respectively, over a $40^{\circ} \times 60^{\circ}$ (potentially $60^{\circ} \times 80^{\circ}$ ) large FOV.

\section{References}

[1] T. Sandner et al., 3D imaging using resonant large-aperture MEMS mirror arrays..., Proc. Optical MEMS \& Nanophotonics (2008) 78 - 79

[2] H. Wölfelschneider et al., Schnelle Entfernungsmessung für Laserscanner, Technisches Messen: ATM, TM 72, (7-8), (2005) 455-467

[3] T. Sandner, M. Wildenhain et al., Large aperture MEMS scanner module for 3D distance measurement, SPIE 7594, (2010) pp. 75940D-1-11

[4] J. Thielemann, T. Sandner et al., TACO: Threedimensional Camera with Object Detection and Foveation, (2010) 1-4, EC FP7 grant no 248623

[5] H. Schenk, T. Sandner et al. Single crystal silicon micro mirrors, Phys. Status Solidi C, (2009), 1-8

[6] T. Sandner, D. Jung, et al., Microscanner with Vertical out of Plane Combdrive, IEEE/LEOS Proc. Optical MEMS \& Nanophotonics, (2011)

[7] D. Jung, T. Sandner et al., Vertical comb drive microscanners for beam steering, linear scanning, Proc. SPIE 8252 (2012) doi: 10.1117/12.906690

[8] V. Milanovic et al. Monolithic vertical combdrive actuators, Proc. Optical MEMS (2002) 57-58

[9] W. Piyawattanametha et al., 2D Scanner Driven by Angular Vertical Comb Actuators, J. of MEMS, Vol. 14, No. 6 (2005) 1329-1338

[10] D. Jung et al., Fabrication of 3D Comb Drive..., Proc. SPIE 7208, (2009) 72080A-1--11

[11] S. Britta, C. Baulig et al., Micromirror-based sending and detection optical assembly for timeof-flight laser scanners; Proc. SPIE 8439 (2012)

[12] K. Janschek, T. Sandner et al., Open Loop Control for Quasistatic Microscanners, Proc VDIMechatroniktagung, Aachen (2013). 